\title{
Calidad de vida en las pacientes con incontinencia urinaria
}

\author{
Martínez Córcoles B, Salinas Sánchez AS, Giménez Bachs JM, Donate Moreno MJ, \\ Pastor Navarro H, Virseda Rodríguez JA.
}

Servicio de Urología. Complejo Hospitalario Universitario de Albacete

Actas Urol Esp. 2008;32(2):202-210

\begin{abstract}
RESUMEN
CALIDAD DE VIDA EN LAS PACIENTES CON INCONTINENCIA URINARIA

Objetivo: Valorar la calidad de vida de las pacientes con incontinencia urinaria de esfuerzo (IUE), asistidas en el Servicio de Urologia del Complejo Hospitalario Universitario de Albacete (CHUA).

Método: Entre Noviembre de 2001 y Diciembre de 2005, se han encuestado en nuestro centro a 126 pacientes afectas de IUE, mediante el King's Health Questionnaire (KHQ) (Encuesta de Salud de King), el cual es un instrumento específico para la medida de la calidad de vida en pacientes con incontinencia urinaria.

Resultados: la edad media de las pacientes fue de 57,09 años (DE: 9,57) El índice de masa corporal medio fue de $28,14 \mathrm{Kg} / \mathrm{m}^{2}$ (DE: 4,66). La media de evolución de la incontinencia urinaria fue 114,48 meses, con una mediana de 96 meses. Durante la última semana, un total de 73 mujeres $(57,94 \%)$ tuvieron más de 10 episodios de escape de orina al dia. En cuanto al número de compresas utilizadas diariamente, un 82,5\% (104 casos) utilizaban menos de 6 compresas diarias. Por lo que respecta al diagnostico urodinámico, en 8 casos $(6,3 \%)$ se describió como normal, en $16(12,7 \%)$ fueron diagnosticadas de incontinencia urinaria mixta y, finalmente, $102(81 \%)$ como incontinencia genuina de esfuerzo. La escala mejor puntuada fue la de Relaciones personales con una puntuación media de 26,8, mientras que la escala peor puntuada fue la del Impacto de la incontinencia urinaria, con una puntuación media de 82,96. Se comprobó que en líneas generales las puntuaciones más bajas (mejor calidad de vida) se obtuvieron en los grupos de edad más jóvenes. Las puntuaciones en las escalas de Salud general, Limitación en las actividades de la vida diaria, Limitaciones de la actividad fisica, Limitaciones de la actividad social y en la de Emociones, fueron significativamente superiores en pacientes que habian sido sometidas previamente a histerectomia. Las pacientes con mayor tiempo de evolución de su incontinencia urinaria puntuaron más bajo la escala de Relaciones personales ( $r=0,179 ; \mathrm{p}=0,045)$.

Conclusiones: Las pacientes con I.U. tienen afectada su calidad de vida que les limita tanto su actividad fisica como su imagen corporal. Su calidad de vida empeora con la edad, un mayor grado de incontinencia, una mayor sintomatología miccional y cuando se asocian episodios de infección urinaria.
\end{abstract}

Palabras clave: Incontinencia. Calidad de vida. Cuestionario de Salud de King.

\section{ABSTRACT}

QUALITY OF LIFE IN PATIENTS WITH URINARY INCONTINENCE

Objective: To assess the quality of life of female patients with urinary stress incontinence (USI) who were attended at the urology department of the University Hospital at Albacete.

Method: Between November 2001 and December 2005, 126 patients with USI were surveyed in our hospital using the King's Health Questionnaire (KHQ), which is a specific instrument to measure the quality of life among female patients with urinary incontinence (UI).

Results: The mean age of patients was 57.09 años (SD: 9.57) and the mean BMI was $28.14 \mathrm{Kg} / \mathrm{m}^{2}$ (SD: 4.66$)$. The mean evolution of UI was 114.48 months, with a median of 96 months. During the last week of the study period, a total of 73 women $(57.94 \%)$ had more than 10 urine leaks a day. As regards the number of sanitary towels used a day, $82.5 \%$ (104 cases) stated they used less than 6 . The urodynamic diagnosis was described as normal in 8 cases $(6.3 \%), 16$ cases $(12.7 \%)$ were diagnosed as mixed urinary incontinence and, finally, 102 cases $(81 \%)$ suffered genuine USI. The best scores were presented in the Personal Relationships scale with a mean score of 26.8 , whereas the worse scores were noted in that of Impact of Urinary Incontinence, whose mean score was 82.96. Generally, the lowest scores (better quality of life) were obtained in the youngest age groups. Scores in the scales of General Health, Impact on daily activities, Impact on physical activity, Impact on social activity and Emotions, were significantly higher in patients who had undergone a histerectomy. Patients whose evolution of urinary incontinence was longer presented lower scores in the Personal Relationships scale ( $\mathrm{r}=0.179 ; \mathrm{p}=0.045)$.

Conclusions: The quality of life of female patients with U.I. is affected, which limits both their physical activity and image. Their quality of life worsens with age, a greater extent of incontinence, greater urinary symptomatology and when episodes of urinary infection are associated.

Keywords: Incontinence. Quality of Life. King's Health Questionnaire. 
$\mathrm{E}^{\mathrm{n}}$ n 1979, Bates et al. definieron la incontinencia urinaria (I.U.) como la pérdida involuntaria de orina a través de la uretra, que se puede demostrar de forma objetiva y que ocasiona un problema social o de higiene ${ }^{1}$. En 2002, la International Continence Society (ISC) actualizó la terminológica y las definiciones, para poder acordar y utilizar términos idénticos y uniformes en los estados de disfunción del tracto urinario inferior, como la I.U. ${ }^{2}$. Así, se define la incontinencia urinaria de esfuerzo (IUE) como la "pérdida involuntaria de orina que tiene lugar cuando, en ausencia de contracción del detrusor, la presión intravesical supera la presión uretral máxima”.

La I.U. es un problema que afecta aproximadamente al $6 \%$ de la población estadounidense y aunque se estima una prevalencia en los adultos del 15 al 30\%, se presenta en todas las edades, detectándose un incremento progresivo según avanza la edad. Así mismo supone un gran coste, estimado en 10 billones de dólares al año ${ }^{3}$. En España está reconocido como un problema de salud que genera un gran gasto económico a los sistemas sanitarios públicos. En el Área 6 de Madrid, en el año 2000, supuso el 8,8\% del gasto total de farmacia ${ }^{4}$.

El interés por el concepto y la medida de la calidad de vida en el área de la salud surge por varios motivos. Por un lado, el entendimiento de la salud como un estado de completo bienestar físico, psíquico y social, no solamente como la ausencia de enfermedad y dolencia; por otro lado, el extraordinario desarrollo de nuevos productos farmacológicos y de tecnología sanitaria, han propiciado el interés por la medida de la "calidad de vida”, cuyo propósito consiste en proporcionar una evaluación más comprensiva, integral y válida del estado de salud de un individuo o grupo ${ }^{5}$.

Así pues, el bienestar físico y emocional son los dos aspectos más importantes, aunque hay que considerar otros como el sueño, ansiedad, fatiga, depresión, comunicación, relaciones con la pareja, relaciones sociales, etc. ${ }^{6}$. Todas estas dimensiones están claramente afectadas en las pacientes con incontinencia urinaria. Por este motivo nos planteamos determinar la calidad de vida de las mujeres con I.U. de esfuerzo medida mediante un cuestionario específico de calidad de vida en incontinencia como es la Encuesta de Salud de King.

\section{MATERIAL Y MÉTODO}

Se trata de un estudio transversal. Se analizaron los resultados obtenidos mediante el King's Health Questionnaire (KHQ) (Encuesta de Salud de King), el cual es un instrumento específico para la medida de la calidad de vida en pacientes con incontinencia urinaria. Consta de 21 ítems distribuidos en 9 dimensiones: percepción del estado de salud general (1 ítem); impacto de la IU en su vida ( 1 item), (para evitar confusiones estas dos dimensiones, las analizaremos como una única dimensión denominada "Salud general"); limitación en las actividades diarias (2 items); limitaciones sociales ( 2 ítems); limitaciones físicas (2 items); relaciones personales (3 items); emociones (3 ítems); sueño/energía (2 items); impacto de la incontinencia (5 ítems). Cada ítem del KHQ tiene una escala de respuesta tipo Likert con 4 posibles opciones. El rango de puntuaciones de cada dimensión va de 0 (mejor CVRS) a 100 (peor CVRS) ${ }^{7-9}$. Este cuestionario esta diseñado para ser autoadministrado ${ }^{7}$.

El estudio se inició en Noviembre de 2001 y finalizó en Diciembre de 2005. Mediante muestreo consecutivo se seleccionaron un total de 126 pacientes en el Servicio de Urología del Complejo Hospitalario Universitario de Albacete.

Además de la puntuación del cuestionario $\mathrm{KHQ}$, recogieron datos referentes a:

- Tiempo de evolución de la incontinencia (en meses)

- Antecedentes de ITU.

- Frecuencia miccional diurna (en minutos).

- Frecuencia miccional nocturna (número de veces).

- Número de episodios de escapes al día en la última semana.

- Número de compresas que usa al día.

- Presencia de cistocele.

- Grado de cistocele: I-II-III. Grado I: Existe mínima hipermovilidad de la base de la vejiga. Grado II: La base de la vejiga protuye en el introito sin exteriorizarse. Grado III: La vejiga protuye fuera de la vagina con el esfuerzo.

- Urgencia miccional.

- Presencia de incontinencia de urgencia. 
- Informe urodinámico: urodinámica normal, incontinencia pura, incontinencia mixta.

Finalmente también se analizó variables referentes a: edad, indice de masa corporal, presencia de enfermedades crónicas, consumo de medicación crónica referido por los pacientes y antecedentes obstétricos o ginecológicos.

Se realizó un análisis descriptivo de cada una de las variables del estudio además de un análisis bivariante mediante pruebas de independencia para comparar proporciones observadas en grupos independientes (chi-cuadrado), prueba de tendencia lineal de Mantel-Haenscel y para estudiar la relación entre una variable binaria y una cuantitativa, se utilizó la prueba "t" de StudentFisher. En casos de no cumplirse las condiciones de aplicación de todos estos test se utilizaron las correspondientes pruebas no paramétricas.

Mediante regresión lineal se construyeron diferentes modelos para valorar las variables que se asociaron de forma independiente con una mayor puntuación en cada una de las escalas del cuestionario. Ase utilizó el método de inclusión por pasos

\section{RESULTADOS}

La edad media de las 126 mujeres de la muestra fue de 57,09 años con una desviación típica (DE) de 9,57 y un intervalo de confianza al 95\% (IC 95\%) comprendido entre 55,40 y 58,78. La edad mínima fue de 36 años y la máxima de 76 años. El grupo de edad más frecuente correspondió a las pacientes comprendidas entre los 56 y 60 años (20,6\%). El 77,6\% de las mujeres de la muestra presentaron edades comprendidas entre los 46 y los 65 años.

Manifestaron padecer alguna enfermedad de carácter crónico y no relacionada con su patología urológica 88 pacientes $(69,8 \%)$. $\mathrm{El} 42,1 \%$ de las mujeres presentaron solo una enfermedad (53 casos). Las pacientes con alguna enfermedad crónica presentaron una edad media superior a las que no se declararon enfermas: 58,47 frente a 53,89 años $(\mathrm{p}=0,013)$.

Estaban tomando algún tipo de medicación no relacionado con tratamientos médicos de su incontinencia urinaria 85 mujeres $(67,5 \%)$. El $40,5 \%$ de las mujeres utilizaban un solo fármaco (51 mujeres). Los fármacos más frecuentemente utilizados fueron los hipotensores $(41,18 \%)$ seguido de los antiinflamatorios no esteroideos (AINEs) $(27,06 \%)$.

$\mathrm{El}$ indice de masa corporal (IMC) medio fue de $28,14 \mathrm{Kg} / \mathrm{m}^{2}$ (DE: 4,66; IC 95\%: 27,32-28,96), con un rango entre 20,03 y $42,87 \mathrm{Kg} / \mathrm{m}^{2}$. Un $42,9 \%$ de las mujeres (54 casos) presentaron sobrepeso (IMC entre 25 y $30 \mathrm{Kg} / \mathrm{m}^{2}$ ) y un $30,2 \%$ (38 casos) presentaron un IMC en rango de obesidad $\left(>30 \mathrm{Kg} / \mathrm{m}^{2}\right)$

En el momento del estudio, 96 mujeres $(76,2 \%)$ estaban menopausicas, con una edad media de inicio de ésta a los 46,09 años (DE: 5,34; IC 95\%: 42,51\%-49,68).

Tuvieron antecedentes de histerectomía 32 mujeres $(25,4 \%)$, realizándose ésta por vía abdominal en $26(81,25 \%)$ y en el resto de casos por vía vaginal. Por otro lado, 13 pacientes (10,3\%) habían sido sometidas a doble anexectomía, a una edad media de 48,82 años (DE: 8,59; IC 95\%: 43,05-59,59). Finalmente, 7 pacientes $(5,6 \%)$ llevaban tratamiento sustitutivo con estrógenos.

La media de evolución de la incontinencia urinaria fue 114,48 meses, con una mediana de 96 meses. En relación a la frecuencia miccional diurna, 76 pacientes orinaban cada 60-120 minutos $(60,3 \%)$. En cuanto a la frecuencia miccional nocturna, 33 pacientes se levantaban a orinar durante la noche 3 veces $(26,2 \%)$.

Durante la última semana, un total de 73 mujeres $(57,94 \%)$ tuvieron más de 10 episodios de escape de orina al día, mientras que únicamente 4 presentaron un solo episodio de incontinencia al día $(3,2 \%)$. En cuanto al número de compresas utilizadas diariamente, un 82,5\% (104 casos) utilizaban menos de 6 compresas diarias. Urgencia miccional e incontinencia urinaria de urgencias se evidenció en 18 ocasiones respectivamente $(14,3 \%)$.

No presentaban ningún grado de cistocele 38 pacientes $(30,2 \%)$ y del resto de mujeres con cistocele, en 61 casos éste fue de grado III $(48,4 \%)$; en 25 de grado II $(19,8 \%)$ y en 2 de grado I $(1,6 \%)$.

Referían haber presentado algún episodio de infección urinaria 46 pacientes (36,5\%). La existencia de infección urinaria se asoció con la presencia de cistocele, de tal manera que presentaban antecedentes de infección urinaria un 26,3\% 
de las mujeres sin cistocele frente a un 40,9\% de las mujeres que tenían algún grado de cistocele ( $\mathrm{p}=0,05)$.

Por lo que respecta al diagnostico urodinámico de las pacientes del estudio, en 8 casos $(6,3 \%)$ el estudio urodinámico se describió como normal, en $16(12,7 \%)$ fueron diagnosticadas de incontinencia urinaria mixta y, finalmente, 102 (81\%) fueron catalogadas como portadoras de incontinencia genuina de esfuerzo.

La escala mejor puntuada fue la de Relaciones personales con una puntuación media de 26,89 (IC 95\%: 21,16-32,63), mientras que la escala peor puntuada fue la Impacto de la incontinencia urinaria, con una puntuación media de 82,96 (IC 95\%: 80,15-85,77). En la Tabla I se exponen las puntuaciones medias para cada una de las escalas de KHQ.

La edad de las pacientes únicamente se correlacionó débilmente con la escala de Sueño/Energía, de forma que las pacientes más jóvenes puntuaron más bajo esta escala (mejor calidad de vida) y viceversa ( $r=0,268 ; p=0,002)$.

Aunque no se encontró relación significativa en cuanto a las puntuaciones medias de cada una de las escalas con los distintos grupos de edad analizados, sí que se comprobó que en líneas generales las puntuaciones más bajas (mejor calidad de vida) se obtuvieron en los grupos de edad más jóvenes. En la Figura 1 se puede comprobar cómo existe una tendencia a puntuaciones medias más altas (peor calidad de vida) según aumenta la edad de los pacientes, circunstancia común en cada una de las escalas del cuestionario KHQ.

Las puntuaciones de las diversas escalas de cuestionario KHQ no se asociaron con la existen-

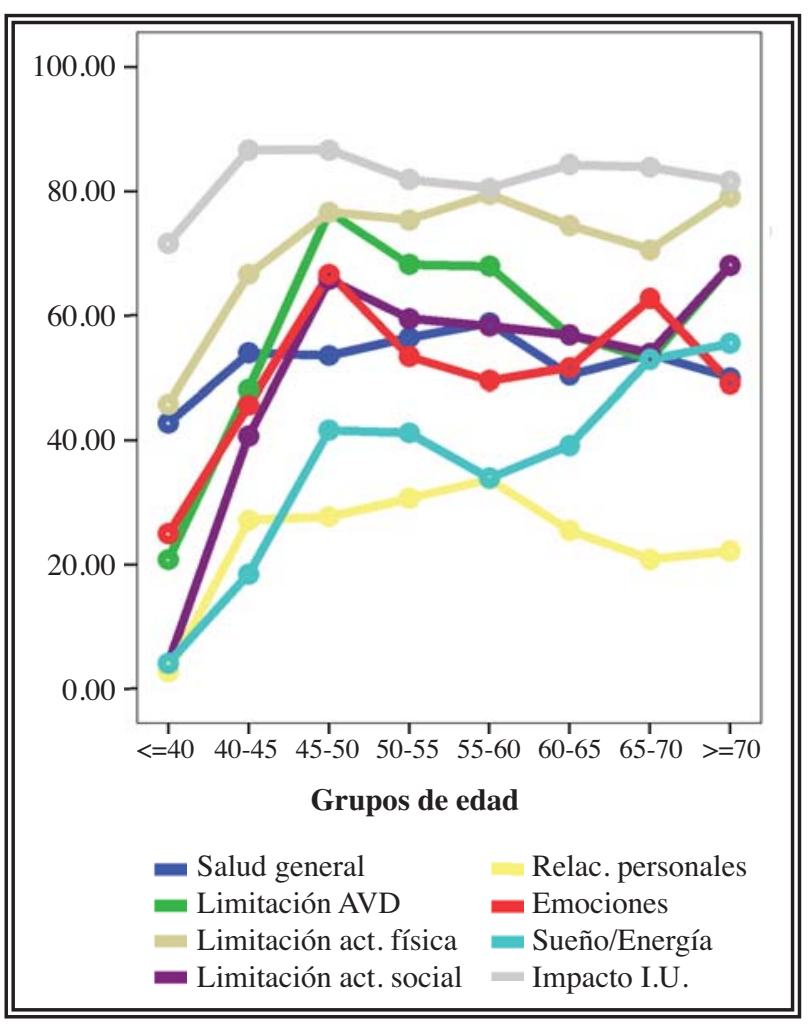

FIGURA 1. Puntuaciones medias para cada escala del cuestionario KHQ para cada grupo de edad.

cia de antecedentes de enfermedades crónicas a excepción de la escala de Relaciones personales, de manera que los pacientes con enfermedades crónicas asociadas obtuvieron mayores puntuaciones en esta escala: 30,55 (DE: 33,87) frente a 18,42 (DE: 27,73) en los que no tenían enfermedades crónicas $(\mathrm{p}=0,038)$. Igualmente, las pacientes que consumían habitualmente algún tipo de medicación puntuaron significativamente mayor la escala de Relaciones personales: 31,63 (DE: 39,96) frente a 17,07 (DE: 21,11) en los que no utilizaban medicación ( $\mathrm{p}=0,011)$.

Tabla 1. Puntuación media de las escalas KHQ

\begin{tabular}{lcccc}
\hline Escalas & Media (DE) & IC 95\% & Rango & Mediana \\
\hline Salud general & $54,08(18,92)$ & $50,74-57,4$ & $0-100$ & 57,14 \\
Limitación en la actividad diaria & $62,93(35,64)$ & $56,68-69,25$ & $0-100$ & 66,66 \\
Limitación en la actividad física & $74,47(29,64)$ & $69,24-79,70$ & $0-100$ & 83,33 \\
Limitación en la actividad social & $56,88(39,40)$ & $49,93-63,82$ & $0-100$ & 58,33 \\
Relaciones personales & $26,89(32,51)$ & $21,16-32,63$ & $0-100$ & 11,11 \\
Emociones & $53,88(36,38)$ & $47,46-60,29$ & $0-100$ & 44,44 \\
Sueño/Energía & $39,68(35,89)$ & $33,35-46,01$ & $0-100$ & 33,33 \\
Impacto de la I.U. & $82,96(15,92)$ & $80,15-85,77$ & $26,67-100$ & 86,66 \\
\hline
\end{tabular}


Las puntuaciones en las escalas de Salud general, Limitación en las actividades de la vida diaria, Limitaciones de la actividad física, Limitaciones de la actividad social y en la de Emociones, fueron significativamente superiores en pacientes que habían sido sometidas previamente a histerectomía. Igual circunstancia ocurrió con la escala de Emociones en las mujeres que habían sido sometidas a anexectomía previa. En la Tabla II se detallan estos datos. Las pacientes que estaban bajo tratamiento con estrógenos tuvieron puntuaciones más altas en la escala de Salud general: 66,34 (DE: 13,59) frente a 53,30 (DE: 18,94) de las mujeres que no seguian tratamiento sustitutivo $(\mathrm{p}=0,034)$.

Las pacientes con mayor tiempo de evolución de su incontinencia urinaria puntuaron más bajo la escala de Relaciones personales $(r=0,179 ; p=0,045)$. Las puntuaciones en todas las escalas del KHQ presentaron correlaciones positivas en intensidad variable entre débil y moderadas con el número de episodios de escapes urinarios durante la última semana y con el número de compresas que necesitaban las pacientes. Las escalas de Limitación de actividad social, Relaciones personales y Sueño/Energía obtuvieron mayores puntuaciones en función de la frecuencia miccional nocturna. Por lo que respecta a las puntuaciones del KHQ y la frecuencia miccional diurna, se comprobó que las pacientes con un intervalo de tiempo entre micciones mayor puntuaron más bajo la escala de Sueño/Energía ( $r=-0,275 ; p=0,002)$. La Tabla III muestra los coeficientes de correlación entre las escalas del KHQ y la frecuencia miccional nocturna, número de escapes de orina/día y número de compresas/día durante la última semana. Finalmente, ni la existencia y anexectomizadas última semana de urgencia miccional ni la presencia de incontinencia urinaria de urgencia asociada a la incontinencia urinaria de esfuerzo se asociaron con las puntuaciones del cuestionario KHQ.

Las pacientes con algún grado de cistocele puntuaron significativamente más bajo $(48,61$; DE: 35,87) la escala de Emociones en relación a las que no presentaban ningún grado de cistocele $(66,08$; DE: 35,04) ( $\mathrm{p}=0,013)$. Entre las pacientes con cistocele no se encontraron diferencias en relación a las puntuaciones del KHQ y el grado de cistocele.

Tabla 2. Puntuaciones medias de las escalas KHQ en mujeres histerectomizadas

\begin{tabular}{lccc}
\hline Escala & $\begin{array}{c}\text { Histerectomia } \\
\text { Media (DE) }\end{array}$ & $\begin{array}{c}\text { No histerectomia } \\
\text { Media (DE) }\end{array}$ & p \\
\hline Salud general & $62,05(15,65)$ & $51,36(19,23)$ & 0,005 \\
Limitación de AVD & $72,92(30,45)$ & $59,57(36,78)$ & 0,047 \\
Limitación de actv. fisica & $86,45(20,05)$ & $70,39(31,31)$ & $<0,001$ \\
Limitación de actv. social & $70,31(35,60)$ & $52,30(39,75)$ & 0,02 \\
Emociones & $70,13(36,30)$ & $48,34(34,90)$ & 0,005 \\
\hline Escala & Anexectomia & No anexectomia & $\mathbf{p}$ \\
& Media (DE) & Media (DE) & \\
\hline Emociones & $76,07(30,38)$ & $51,32(36,25)$ & 0,021 \\
\hline
\end{tabular}

Tabla 3. Coeficientes de correlación entre las escalas del KHQ y frecuencia miccional nocturna. Número de escapes/día y número de compresas/día durante la

\begin{tabular}{|c|c|c|}
\hline \multicolumn{3}{|c|}{ Frecuencia miccional nocturna } \\
\hline ESCALA & $\mathbf{r}$ & $\mathbf{p}$ \\
\hline Limitación de actividad social & 0,258 & 0,04 \\
\hline Relaciones personales & 0,215 & 0,016 \\
\hline Sueño/Energía & 0,420 & $<0,001$ \\
\hline \multicolumn{3}{|c|}{ Número de escapes/dia } \\
\hline Escala & $\mathbf{r}$ & $\mathbf{p}$ \\
\hline Salud general & 0,318 & $<0,0001$ \\
\hline Limitación de AVD & 0,409 & $<0,0001$ \\
\hline Limitación de actividad física & 0,431 & $<0,0001$ \\
\hline Limitación de actividad social & 0,487 & $<0,0001$ \\
\hline Relaciones personales & 0,264 & 0,003 \\
\hline Emociones & 0,392 & $<0,0001$ \\
\hline Sueño/Energía & 0,244 & 0,006 \\
\hline Impacto de la I.U. & 0,449 & $<0,0001$ \\
\hline \multicolumn{3}{|c|}{ Número de compresas/dia } \\
\hline Escala & $\mathbf{r}$ & $\mathbf{p}$ \\
\hline Salud general & 0,273 & 0,002 \\
\hline Limitación de AVD & 0,311 & $<0,0001$ \\
\hline Limitación de actividad física & 0,327 & $<0,0001$ \\
\hline Limitación de actividad social & 0,409 & $<0,0001$ \\
\hline Relaciones personales & 0,184 & 0,039 \\
\hline Emociones & 0,295 & $<0,001$ \\
\hline Sueño/Energía & 0,281 & $<0,001$ \\
\hline Impacto de la I.U. & 0,360 & $<0,0001$ \\
\hline
\end{tabular}


Las variables que mostraron una asociación estadísticamente significativa con una mayor puntuación en la escala de Salud general fueron: un mayor número de compresas utilizadas durante la última semana, la ausencia de cistocele, un mayor índice de masa corporal, una menor edad de aparición de la menopausia y la necesidad de seguir tratamiento con estrógenos. La variabilidad explicada por este modelo fue del $30,9 \%\left(r^{2}=0,309\right)(\mathrm{F}=6,641 ; \mathrm{p}<0,0001)$.

Las variables que mostraron una asociación estadísticamente significativa con una puntuación mayor en la escala de Limitación de actividad de la vida diaria fueron: presencia de antecedentes de cirugía abdominal, mayor número de escapes de orina durante la última semana. La variabilidad explicada por este modelo fue del $31,4 \%\left(r^{2}=0,314\right)(\mathrm{F}=10,424 ; \mathrm{p}<0,0001)$.

Por lo que respecta a la escala de Limitación de actividad física, las variables que mostraron una asociación estadísticamente significativa con una puntuación mayor en esta escala fueron: un mayor número de escapes de orina al día durante la última semana, la existencia de antecedentes de cirugía. La variabilidad explicada por este modelo fue del $32 \%\left(\mathrm{r}^{2}=0,320\right)(\mathrm{F}=14,402 ; \mathrm{p}<0,0001)$.

En cuanto a la escala de Limitaciones en la actividad social, las variables que mostraron una asociación estadísticamente significativa con una puntuación mayor en esta escala fueron: un mayor número de escapes de orina al día durante la última semana, un mayor número de compresas. La variabilidad explicada por este modelo fue del 37,8\% $\left(r^{2}=0,378\right)(F=18,649 ; p<0,0001)$.

La escala de Relaciones personales obtuvo puntuaciones más altas de forma estadísticamente significativa en las pacientes que necesitaban tratamiento médico habitual, tenían antecedentes de cirugía abdominal, presentaban un mayor índice de masa corporal. La variabilidad explicada por este modelo fue del $26,8 \%\left(r^{2}=0,268\right)(F=8,314$; $\mathrm{p}<0,0001)$.

Una mayor puntuación en la escala de Emociones se asoció de forma estadísticamente significativa con un mayor número de escapes de orina durante la última semana, la necesidad de tratamiento sustitutivo con estrógenos, una menor edad de aparición de la menopausia y la ausencia de cistocele. La variabilidad explicada por este modelo fue del 32,1\% $\left(\mathrm{r}^{2}=0,321\right)(\mathrm{F}=10,753 ; \mathrm{p}<0,0001)$.
En la escala de Sueño/Energía, las variables asociadas de forma estadísticamente significativa con una mayor puntuación fueron: una mayor frecuencia miccional nocturna, la ausencia de cistocele, la presencia de antecedentes de infecciones urinaria, un mayor número de escapes durante la última. La variabilidad explicada por este modelo fue del $30,5 \%\left(r^{2}=0,305\right)(F=7,904$; $\mathrm{p}<0,0001)$.

Finalmente, las puntuaciones mayores en la escala de Impacto de la incontinencia urinaria se asociaron de forma estadísticamente significativa a la presencia de un mayor número de escapes de orina durante la última semana y a la necesidad de utilizar un mayor número de compresas al día. La variabilidad explicada por este modelo fue del $26,8 \%\left(r^{2}=0,268\right)(\mathrm{F}=17,025 ; \mathrm{p}<0,0001)$.

En la Tabla IV se exponen las variables asociadas en el modelo de regresión múltiple con las distintas escalas del cuestionario KHQ.

\section{DISCUSIÓN}

El KHQ se eligió porque a diferencia de otros cuestionarios genéricos (que incluyen dimensiones genéricas de la CVRS y son poco sensibles a los cambios clínicos), es un cuestionario específico, que no sólo mide los síntomas urinarios sino que además mide el impacto de la I.U. en las áreas física, social y mental. Su contenido, robustez psicométrica y su relativa simplicidad son factores que facilitan su utilización ${ }^{7}$. La validación de este cuestionario corrió a cargo de Xavier Badía et al. ${ }^{7}$.

La I.U. es una patología frecuente, que alcanza alrededor del 40\% de la población femenina en España, con un incremento de la prevalencia, conforme avanza la edad ${ }^{10}$. Es evidente que la incidencia y la prevalencia de la I.U. se incrementan con la edad además de con otros factores. Así, como causa en la que influye directamente la edad tenemos los cambios postmenopáusicos como consecuencia de la deprivación estrogénica, condición que está relacionada con el riesgo de padecer incontinencia urinaria de esfuerzo $^{10,11}$.

Actualmente, los estudios epidemiológicos existentes en diversos estudios valoran la incidencia y la prevalencia de la I.U. con uniformidad y cuyos resultados son comparables entre ellos. 
Tabla 4. Variables asociadas mediante regresión múltiple a las escalas del cuestionario KHQ

\begin{tabular}{|c|c|c|c|}
\hline Variable & $\begin{array}{r}\text { Salud general } \\
\text { Coeficiente }\end{array}$ & IC $95 \%$ & $\mathbf{p}$ \\
\hline Número compresas/día & 1,759 & $0,429 / 3,089$ & 0,01 \\
\hline Îndice de masa corporal & 0,753 & $0,031 / 1,475$ & 0,041 \\
\hline Edad de la menopausia & $-0,701$ & $-1,276 /-0,137$ & 0,016 \\
\hline Cistocele & $-8,265$ & $-16,020 /-0,509$ & 0,037 \\
\hline Constante & 65,958 & $29,773 / 102,142$ & $<0,0001$ \\
\hline \multicolumn{4}{|c|}{ Limitación de la actividad de la vida diaria } \\
\hline Variable & Coeficiente & IC $95 \%$ & $\mathbf{p}$ \\
\hline Antecedentes cirugía abdominal & 21,480 & $9,289 / 33,671$ & 0,001 \\
\hline Número de escapes de orina/día & 1,431 & $0,403 / 2,459$ & 0,007 \\
\hline Constante & 24,311 & $6,121 / 42,500$ & $<0,0001$ \\
\hline \multicolumn{4}{|c|}{ Limitación de la actividad física } \\
\hline Variable & Coeficiente & IC $95 \%$ & $\mathbf{p}$ \\
\hline Número de escapes orina/día & 1,908 & 1,037 / 2,778 & $<0,0001$ \\
\hline Antecedentes cirugía abdominal & 13,971 & 3,699 / 24,243 & 0,008 \\
\hline Constante & 48,446 & $38,334 / 58,558$ & $<0,0001$ \\
\hline \multicolumn{4}{|c|}{ Limitación de la actividad social } \\
\hline Variable & Coeficiente & IC $95 \%$ & $\mathbf{p}$ \\
\hline Número de escapes orina/día & 2,493 & $1,991 / 3,795$ & $<0,0001$ \\
\hline Número de compresas/día & 4,069 & 1,059 / 7,079 & 0,009 \\
\hline Constante & 14,201 & $0,387 / 28,015$ & 0,044 \\
\hline \multicolumn{4}{|c|}{ Relaciones personales } \\
\hline Vartaivic & & $1050 \%$ & $\mathbf{p}$ \\
\hline Uso de medicación habitual & 24,797 & $11,599 / 37,994$ & $<0,0001$ \\
\hline Antecedente cirugía abdominal & 16,466 & $4,372 / 28,560$ & 0,008 \\
\hline Índice de masa corporal & 1,556 & $0,272 / 2,840$ & 0,018 \\
\hline Constante & $-60,255$ & $-99,338 /-21,173$ & 0,003 \\
\hline \multicolumn{4}{|c|}{ Emociones } \\
\hline Número de escapes orina/día & 2,016 & $0,893 / 3,140$ & 0,001 \\
\hline Cistocele & $-29,035$ & $-43,669 /-14,402$ & $<0,0001$ \\
\hline Edad de la menopausia & $-1,263$ & $-2,381 /-0,145$ & 0,027 \\
\hline Tratamiento con estrógenos & 26,579 & $1,710 / 51,448$ & 0,036 \\
\hline Constante & 114,856 & $55,741 / 173,97$ & $<0,0001$ \\
\hline \multicolumn{4}{|c|}{ Sueño/Energía } \\
\hline Frecuencia miccional nocturna & 6,663 & 2,615 / 10,710 & 0,002 \\
\hline Cistocele & $-18,037$ & $-32,268 /-3,807$ & 0,014 \\
\hline Antecedentes de infección urinaria & 17,336 & $4,071 / 30,601$ & 0,011 \\
\hline Número de escapes orina/día & 1,315 & $0,225 / 2,405$ & 0,019 \\
\hline Constante & 23,289 & $4,041 / 42,537$ & 0,018 \\
\hline \multicolumn{4}{|c|}{ Impacto de la incontinencia urinaria } \\
\hline Número de escapes orina/día & 0,743 & $0,168 / 1,318$ & 0,012 \\
\hline Número de compresas/día & 1,784 & $0,457 / 3,110$ & 0,009 \\
\hline Constante & 67,813 & $61,866 / 73,761$ & $<0,0001$ \\
\hline
\end{tabular}

Así, en España, la prevalencia oscila desde el $31,7 \%$ al $53,3 \%$ conforme se incrementa la edad ${ }^{10,12}$.

Observando los datos obtenidos de nuestras pacientes diagnosticadas de incontinencia de orina en espera de tratamiento quirúrgico, vemos que la dimensión puntuada más bajo y por tanto con mejor calidad de vida, fue la de Relaciones personales y la más alta y por tanto con peor calidad de vida, fue la dimensión de Impacto de la incontinencia urinaria; siguiéndole en peor calidad de vida la escala de Limitación en la actividad física. Estos datos pueden considerarse lógicos si se tiene en cuenta que en la dimensión de Relaciones personales se valora, fundamentalmente, el entorno familiar y las relaciones de pareja, situaciones que han sido consolidadas a través del tiempo. La dimensión de Impacto de la I.U., fue la peor puntuada y en ello influye el hecho que a las pacientes les incomoda mucho el tener que llevar compresas o pañales de forma más o menos continuada, la preocupación por si huelen mal, etc. También puntuaron mal la escala de Limitación de actividad física por la posible incidencia que la I.U. tiene sobre actividades como hacer gimnasia, algún tipo de deporte, viajar y en general realizar alguna actividad física que pueda provocar un aumento de su escape de orina.

En líneas generales, analizando distintos estudios se puede comprobar que la cali- 
dad de vida se resiente significativamente en las pacientes con incontinencia urinaria y sobre todo afecta al rol emocional, causando depresión en muchos casos. También afecta al sueño, a la vitalidad y limita las actividades sociales 13-15.

En USA, en el año 2004 fueron estudiadas 919 pacientes con vejiga hiperactiva de 54 años de edad media, comprobándose que la incontinencia urinaria estaba relacionada negativamente con la calidad de vida. Se les aplicó el cuestionario SF-36, observando que los parámetros que más se alteraban eran la limitación social, las emociones y la salud mental ${ }^{16}$. Otro estudio realizado en 2005 en pacientes con incontinencia urinaria comprendidas entre 65 y 74 años utilizando el mismo cuestionario, observó resultados similares respecto a las alteraciones en la calidad de vida ${ }^{13}$.

En nuestros resultados observamos que las puntuaciones más bajas (mejor calidad de vida) se dieron en pacientes más jóvenes y que conforme avanzaba la edad las puntuaciones en términos generales eran más altas, por tanto a mayor edad, peor calidad de vida. En la literatura encontramos relación entre peor calidad de vida conforme avanza la edad ${ }^{12,17,18}$. Pero también hay estudios al contrario que a menor edad peor calidad de vida; en este caso hay que matizar que empeoran solamente ciertos aspectos como son la actividad sexual y tensión psicológica ${ }^{19}$, aspectos que no fueron valorados específicamente en nuestro estudio.

La existencia de enfermedades crónicas se relacionó con una puntuación más alta en la escala de Relaciones personales. Igualmente hubo relación entre tomar medicación habitual y la escala de Relaciones personales, así estas pacientes tenían peor calidad de vida que las que no tomaban medicación. Este resultado es paradójico puesto que se supone que no debe influir, el tomar o no medicación, con dicha dimensión que hace referencia al entorno familiar y sexual de la pareja. Paradójicamente el número de enfermedades crónicas no se correlacionó con una peor calidad de vida en la escala de Salud general.

Respecto a los antecedentes ginecológicos, encontramos que las puntuaciones de las escalas de Salud general, Limitación en la actividad diaria, Limitación de la actividad física, Limitación de la actividad social y en la de Emociones, fueron apreciablemente superiores en pacientes que habían sido sometidas a histerectomía. Un estu- dio sobre calidad de vida realizado a 1.249 mujeres a las cuales se les había efectuado una histerectomía ${ }^{20}$ demostró que su calidad de vida se resentía en casi todas las facetas de la actividad diaria y salud mental.

Llama la atención el hecho de que las pacientes que llevaban más tiempo de evolución con su incontinencia urinaria puntuaron más bajo en la escala de Relaciones personales. La explicación podría deberse a que éstas se han habituado a su problema y por tanto esta situación influye poco en su entorno social y familiar.

El número de escapes urinarios durante la última semana y el número de compresas necesarias se correlacionó positivamente y en intensidad variable entre débil y moderada con todas las escalas de cuestionario. Tuvieron mayor puntuación las escalas de Limitación de actividad social, Relaciones personales y Sueño/Energía en función de la frecuencia miccional nocturna. Asímismo las puntuaciones más bajas fueron para la escala de Sueño/Energía, relacionada con el mayor intervalo entre las micciones diurnas. En términos generales, las pacientes refieren peor calidad de vida cuanto mayor es el número de escapes, lo cual les obliga a cambiarse de compresa con más frecuencia y les limita su actividad social. Cuantas más veces se tienen que levantar por la noche a orinar peor duermen y se resiente la dimensión Sueño/ Energía. Por el contrario cuanto más espaciadas están las micciones mejor descansan y, por tanto, mejor puntúan la dimensión Sueño/ Energía.

Puntuaban mejor la escala de Emociones las pacientes que presentaban algún grado de cistocele respecto a las que no lo tenían. No encontramos explicación del porqué las pacientes con algún grado de cistocele tienen mejor calidad de vida en esta escala que las que no lo tienen.

El diagnóstico urodinámico no se correlacionó con ninguna de las escalas del cuestionario $\mathrm{KHQ}$. Aunque nosotros no hemos encontrado dicha correlación, revisando la literatura sí que se encuentran estudios que relacionan ciertos aspectos de la calidad de vida con el diagnóstico urodinámico, aunque dichos estudios son contradictorios. Así pues, un estudio realizado en Noruega analizó la calidad de vida en mujeres con incontinencia urinaria utilizando el cuestionario "Sickness Impact Profile" (Perfil del Impacto de la Enfermedad) a dos grupos, uno de 36 mujeres entre 40 y 60 años 
y otro grupo de 40 mujeres de 70 ó más años; se hicieron dos grupos atendiendo a si padecían incontinencia de estrés o incontinencia de urgencia. Se constató que la calidad de vida se alteraba notablemente en distintas facetas como son: sueño/energía, comportamiento emocional, movilidad, actividades sociales, etc. También se apreció que la calidad de vida se alteraba más en el grupo de mujeres con incontinencia de estrés (7\% vs $17 \%)^{21}$.

Sí que se encontró que las pacientes que habían tenido antecedentes de infección urinaria puntuaron más alto las escalas de Salud general, Sueño/Energía e Impacto de la incontinencia urinaria. Todo esto puede estar en función del aumento del síndrome miccional, mayor número de compresas mojadas, polaquiuria y sobre todo la nicturia que aparece en situación de ITU y que lógicamente incide en una peor calidad de vida.

En definitiva, diversos aspectos pueden influir en las distintas dimensiones de la calidad de vida en pacientes con I.U. Sin embargo, al analizar los resultados de nuestro análisis multivariante, un mayor número de episodios de escape de orina al día y un mayor número de compresas ó pañales utilizados, son los factores que se relacionan con una peor calidad de vida en prácticamente todas las escalas del KHQ, lo cual viene a explicar lógicamente que a mayor gravedad en estos sintomas, el impacto en la calidad de vida es mayor.

Podemos concluir este trabajo diciendo que las pacientes con I.U. tienen afectada su calidad de vida sobre todo en dos aspectos, uno limitando su actividad física a la hora de hacer cualquier actividad y el otro es el impacto que ejerce dicha I.U. en su vida por las posibles implicaciones que ésta ejerce sobre su imagen corporal (uso de empapadores, mala olor corporal etc.). Así mismo la calidad de vida de las pacientes con incontinencia empeora conforme avanza la edad de las mismas, un mayor grado de incontinencia, mayor sintomatología miccional y cuando se asocian episodios de infección urinaria.

\section{REFERENCIAS}

1. Bates P, Bradley WE, Glen E, Griffiths D, Melchior H, Rowan D, et al. The standardization of terminology of lower urinary tract function. J Urol. 1979;121(5):551-554.

2. Abrams P, Cardozo L, Fall M, Griffiths D, Rosier P, Ulmsten U, et al. The standardisation of terminology in lower urinary tract function: report from the standardisation sub-committee of the International Continence Society. Urology. 2003; 61(1):37-49.

3. Galmés Belmonte I, Allona Almagro A, Fernández Fernández E. Anatomía funcional del tracto urinario inferior. En: Cirugia Uroginecológica. Tema Monográfico LXIV Congreso Nacional de Urología. 1999;15-29.
4. Puente Dominguez JL. Sistema Genitourinario. En: Anatomía Humana. Orts Llorca F. Ed. Cientifico-Médica. Madrid 1972;645-52.

5. Reig A, Bordes P. La calidad de vida en la atención sanitaria. En: Tratado de Epidemiología Clínica. Madrid: DuPont Pharma, 1994; pp:327-343.

6. Penson DF, Litwin MS. Calidad de vida relaciona con la salud en pacientes con cánceres urológicos. En: A.U.A. (American Urological Association) Update Series. Ed. Española. Barcelona: Medical Trends S.L. Hoechst Marion Roussel 1998; pp: 29-34.

7. Badia Llach X, Castro Díaz D, Conejero Sigraés J. Validez del cuestionario King s Health para evaluación de la calidad de vida en pacientes con incontinencia urinaria. Med Clin (Barc). 2000;114(17):647-652.

8. Matza LS, Zyczynski TM, Bavendam T. A review of quality-of-life questionnaires for urinary incontinence and overactive bladder: which ones to use and why?. Current Urology Reports. 2004;5(5):336-342.

9. Homma Y, Uemura S. Use of the short form of King's Health Questionnaire to meaure quality of life in patients with an overactive bladder. BJU International 2004; 93:1009-13.

10. Sánchez González R, Ruperez Cordero O, Delgado Nicolás MA, Mateo Fernández R, Hernando Blázquez MA. Prevalencia de incontinencia urinaria en la población mayor de 60 años en atención primaria. Aten Primaria. 1999;24 (7):421-424.

11. Salinas Casado J, Vírseda Chamorro M, Teba del Pino F, Vázquez Alba D, Adot Zurbano JM. Urinay incontinence in menopause. Arch Esp Urol. 2000;53(4):349-354.

12. Peyrat L, Haillot O, Bruyere F, Boutin JM, Bertrand P, Lanson Y. Prevalence and risk factors of urinary incontinence in young women. Prog Urol. 2002;12(1):52-59.

13. Ko Y, Lin SJ, Salmon JW, Bron MS. The impact of urinary incontinence on quality of life of the elderly. Am J Manag Care. 2005;11(4Suppl):S10311.

14. Pang MW, Leung HY, Chan LW, Yip SK. The impact of urinary incontinence on quality of life among women in Hong Kong. Hong Kong Med J. 2005;11(3):158-163.

15. Murillo Mirat J, Cuervo Pinna C, Rodríguez Rincón J. Infecciones urinarias no complicadas. En: Urología: Libro del Residente. L. Resel Estévez. Madrid. 1.998; pp:377-386.

16. Coyne KS, Payne C, Bhattacharyya SK, Revicki DA, Thompson C, Corey $\mathrm{R}$, et al. The impact of urinary urgency and frequency on health-related quality of life in overactive bladder: results from a national community survey. Value Health. 2004;7(4):455-463.

17. Shakhatreh FM. Epidemiology of urinary incontinence in Jordanian women. Saudi Med J. 2005;26(5):830-835.

18. Rohr G, Stovring H, Christensen K, Gaist D, Nybo H, Kragstrup J. Characteristics of middle-aged and elderly women with urinary incontinence. Scand J Prim Health Care. 2005;23(4):203-208.

19. Margalith I, Gillon G, Gordon D. Urinary incontinence in women under 65: quality of life, stress related to incontinence and patterns of seeking health care. Qual Life Res. 2004;13(8):1381-1390.

20. Hartmann KE, Ma C, Lamvu GM, Langenberg PW, Steege JF, Kjerulff KH. Quality of life and sexual function after hysterectomy in women with preoperative pain and depression. Obstet Gynecol. 2004;104(4):701-709.

21. Hunskaar S, Vinsnes A. The quality of life in women with urinary incontinence as measured by the sickness impact profile. J Am Geriatr Soc. 1991;39(4):378-382.

Correspondencia autor: Dr. B. Martínez Córcoles

Servicio de Urología

Complejo Hospitalario Universitario de Albacete

Hermanos Falcó, s/n - 02006 Albacete

Tel.: 967597100

E-mail autor: benjamin_m_c@hotmail.com

Información artículo: Original - Incontinencia urinaria femenina

Trabajo recibido: abril 2007

Trabajo aceptado: mayo 2007 